\title{
A Proposal to Redefine the Thermodynamic Temperature Scale: with a Parable of Measures to Improve Weights
}

\author{
By Prof. W. F. Giauque, University of California
}

$I^{N}$ $\mathrm{N}$ several papers published between 1848 and 1854, Lord Kelvin ${ }^{1}$ proposed the establishment of an absolute scale of temperature, based on Carnot's principle and "quite independent of the physical properties of any specific substance". On such a scale "the absolute values of two temperatures are to one another in the proportion of the heat taken in to the heat rejected in a perfect thermodynamic engine working with a source and a refrigerator at the higher and lower of the temperatures respectively".

The size of the degree used in connexion with Kelvin's thermodynamic scale is arbitrary. The natural way to define the size of the degree is also the easiest way and the one which best enables the widest attainment of accuracy, namely, to select one easily obtained and accurately reproducible fixed point, for example, a melting point, and assign to it a number such that degrees of the desired size are obtained. Unfortunately, this has not been done.

In practice, two fixed points are selected. The temperature interval between the freezing point and the boiling point of water is assigned some definite number of degrees, for example, 100 on the centigrade and 180 on the Fahrenheit scales of temperature. The above two points define the size of the degree and thus the number of degrees between the ice point and the absolute zero. As a consequence of this procedure, the values of the ice point and the boiling point of water on the absolute scale are frequently changed, depending on the experimental observations accepted as being the more reliable.

When an experiment is performed to measure a temperature by means of the fundamental criterion which Kelvin has pointed out, the final result contains not only the experimental error of the measurement itself, but also any error which exists in the particular value of the ice point which may have been adopted as the 'best value'. The value of the ice point on the absolute scale has been the subject of many investigations which certainly must be classed among the more accurate of scientific measurements. Nevertheless, even now the values of the several investigators vary by the larger part of the range $273 \cdot 10^{\circ}$ to $273 \cdot 20^{\circ} \mathrm{K}$., although an intermediate value may be selected which is almost certainly correct within two or three hundredths of a degree.
The only basic temperature scale defined with sufficient completeness to enable the determination of any temperature is the thermodynamic scale. For example, the centigrade scale gives the freezing point and the boiling point of water by definition and nothing more. If it were necessary, for example, to interpolate and extrapolate linearly, by means of such devices as those based on the expansion of liquids and gases, various thermocouples, resistance thermometers, etc., there would be little or no agreement as to the value of a given temperature. As a matter of fact, there is agreement only because the thermodynamic criterion of Kelvin is used in conjunction with the one hundred degree centigrade interval. Interpolation or extrapolation by means of the ideal gas law happens to be a convenient special case of the thermodynamic method.

The basic equations of gas thermometry may be written as :

$$
T_{0^{\circ} \mathrm{C} .}=\frac{100}{\left[(P \bar{V})_{100^{\circ} \mathrm{C} .} /(\bar{P} V)_{0^{\circ} \mathrm{C} .}\right]-1},
$$

and

$$
T=T_{0^{\circ} \mathrm{C}} \frac{(P V)_{T}}{(P V)_{0^{\circ} \mathrm{C}}}
$$

where, in each case, $P V$ is the product of gas pressure and volume corrected for gas imperfection. The T's refer to the absolute temperatures.

In Equation 1 the per cent error in $T_{0^{\circ} \mathrm{C}}$. is 3.73 times that in the experimental ratio


imposed on the experimental inaccuracy involved in the determination of the ratio $(P V)_{T} /(P V)_{0^{\circ} \mathrm{C}}$. of Equation 2. Moreover, observations at the boiling point of water offer greater difficulties than do those at the melting point of ice, and thus contribute more to the inaccuracy of measurement

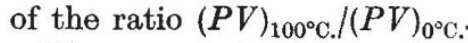

The errors in temperatures on the thermodynamic scale which have their origin in Equation 1 may be eliminated by using the natural method mentioned above, namely, by defining

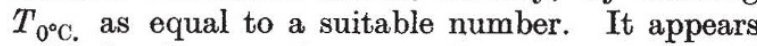
unlikely that a reference point more useful and satisfactory than the ice point can be found.

A proposal of this kind should be advanced only after a careful examination of its consequences. In this connexion, we ask the simple yet significant 
question: Why is it important to know temperatures accurately in terms of any particular scale? For many purposes it would obviously be sufficient to refer temperatures to any arbitrarily adopted scale ; for example, this would suffice to permit the reproduction of a desired set of conditions. It would also permit the accurate use of all energy considerations based on the first law of thermodynamics. However, even if one did not care to inquire further into the basic laws of Nature, there is no escape from their consequences, and it would be found difficult and inconvenient to represent certain important properties by simple equations; for example, the gas law would appear complex if data were obtained in terms of a linear interpolation on a thermometer based on the expansion of a liquid.

The insurmountable objection to an entirely arbitrary scale became evident when Clausius and Kelvin discovered the wide implications of the second law of thermodynamics, which requires the use of a temperature based on Carnct's principle. The use of this temperature leads to the simplest possible representations of all natural phenomena. In fact, all other temperature scales are forced to be as consistent as possible with the thermodynamic scale or they would long since have fallen into disuse.

In addition to the unnecessary inclusion of the experimental error in Equation 1 in the fundamental thermodynamic scale, there is a very common type of mistake that would be permanently eliminated by defining the value of $T_{0^{\circ} \mathrm{C} \text {. }}$ This may be illustrated by an example. Let us say that Laboratory $A$ uses a value of $T_{0^{\circ} \mathrm{C} .}=273 \cdot 19^{\circ} \mathrm{K}$., whereas Laboratories $B$ and $\mathrm{C}$ have decided that $T_{0^{\circ} \mathrm{C} .}=273 \cdot 13^{\circ} \mathrm{K}$. Each of these laboratories makes a determination of the boiling point of oxygen. Their results for the actual observed quantity, $(P V)_{T} /(P V)_{0^{\circ} \mathrm{C}}$, are in exact agreement. $A$ finds $T=90 \cdot 16^{\circ} \mathrm{K}$. but reports it as $-183.03^{\circ} \mathrm{C}$. whereas $B$ and $C$ find $T=90 \cdot 14^{\circ} \mathrm{K}$. and each reports $-182 \cdot 99^{\circ} \mathrm{C}$. A calculation is carried out in a fourth laboratory that favours $T_{0^{\circ} \mathrm{C} .}=273.19$ but the calculator accepts the value $-182.99^{\circ} \mathrm{C}$. because two observers have given it. As is practically always the case, the absolute temperature is required. The calculator obtains it incorrectly by adding 273.19 and -182.99 , which gives $90 \cdot 20^{\circ} \mathrm{K}$. in place of the correct $90 \cdot 16^{\circ} \mathrm{K}$.

The calculator may be accused of inexcusable carelessness, but it is quite probable that the literature failed to specify the reference $T_{0}{ }^{8} \mathrm{C}$. and abstractors are almost unanimously negligent in this respect. By way of analogy, the point we wish to make is that it is more sensible to remove a wire which circumstance has stretched across a path rather than to continue avoiding it by what often amounts to the expenditure of considerable time. This type of confusion caused by emphasis given to the centigrade temperature scale approaches its limit when one laboratory may report an observation at $-273 \cdot 18^{\circ} \mathrm{C}$., whereas another laboratory says there are only $273 \cdot 13$ degrees below $0^{\circ} \mathrm{C}$.

It is not our purpose here to discuss the various observed values of $T_{0^{\circ} \mathrm{C}}$, but as mentioned above it appears probable that a value can be selected which will not differ from the true value by more than two or three hundredths of a degree. Thus a $T_{0}$ can be selected which will not disagree with the centigrade scale by more than one hundredth of one per cent. We know of no thermodynamic observations which are accurate enough to require a correction if a 'best value' of $T_{0^{\circ} \mathrm{C}}$ should be selected to define permanently the absolute scale of temperature. Thus no corrections to any existing literature would be necessitated merely because a best value of $T_{0^{\circ} \mathrm{C}}$ is adopted permanently.

It is not suggested that the centigrade scale be discarded, although, since its main advantages would appear to be only traditional and to permit the use of one less digit in representing ordinary temperatures, it might be desirable to substitute a scale with degrees defined as above but with the ice point taken as zero. At most, the difference between the boiling point and the freezing point on the two scales would not be found to differ by more than one hundredth of a degree, and until at least one experimenter succeeds in making thermodynamic measurements with an accuracy exceeding 0.01 per cent the difference may be ignored. If it should become necessary to make corrections for a few measurements of extraordinary accuracy, it would be a comparatively simple matter to do so.

It should also be made clear that while the thermodynamic criterion is regarded as basic, there is no suggestion of discarding such valuable secondary reference standards as resistance thermometers. It is obviously impractical to measure small temperature differences as accurately by direct observation with a gas thermometer as with a resistance thermometer. For example, a difference of $0.01^{\circ} \mathrm{C}$. is near the limit of error with a gas thermometer, but it can be determined with considerable precision by many other thermometric devices. Nevertheless, it should be kept in mind that it cannot be proved that such useful devices correctly denote what they are assumed to represent except by comparison with the thermodynamic scale. It is hoped that this presentation of the above proposal will initiate wide discussion leading to appropriate action for its general adoption. 
To summarize : The permanent adoption of the best value of the ice point, $T_{0^{\circ} \mathrm{C} \text {. }}$, on the absolute temperature scale would:

(1) Permit an increase in the accuracy with which temperatures may be fixed on the thermodynamic scale.

(2) Eliminate confusion which results from lack of agreement as to the 'best value' of $T_{0^{\circ} \mathrm{C} \text {. and }}$ which would still result if there were occasional changes in a generally adopted 'best value'.

(3) Not in itself necessitate any changes in published thermodynamic data.

In order to emphasize the existing status of temperature scales, we will present the following account of the scientific method which will be entitled :

\section{A Parable of Measures to Improve Weights}

An early scientist, who deserves much credit, desired a standard of weight. He selected two convenient stones. The lighter stone was very smooth, hard and dense, and gave every indication of durability; the heavier stone was definitely of lower quality.

Now the accumulation of information available to this scientist being much less than that of the present, he failed to see the matter with as much clarity as might reasonably be expected of his current successors. His solution of the problem was to state that the heavier stone weighed 100 convenient units more than the lighter standard, which was taken as the zero of weight.

This system served very well for a time, but presently weights below zero were found to be necessary and the tradesmen scarcely knew whether they were buying or selling when the customers asked for 50 units of bread and minus 10 units of cheese.

A temporary respite from this difficulty was obtained in the following manner. A practical scientist decided to search for the lightest stone to be had. The search took him far and wide and, having failed to see nothing, he returned with a somewhat lighter standard for the new zero of weight. As a heavier standard he decided to use the weight of man, and the difference between the two standards was again defined as a certain number of units.

The new choice of the heavier weight was not a very fortunate one and it was soon decided to return to the former standards, but nevertheless the main feature of the plan was retained by making an approximate comparison of the two scales and defining a new unit. There has always been a diversity of opinion as to the desirability of this new unit; some called it more convenient and others called it less convenient, so we will refer to it as the more-or-less convenient unit. Thus 5 convenient units were found to equal 9 more-or-less convenient units and the old standards came out 32 and 212 more-or-less convenient units, respectively, on the new scale, while the new zero of weight was no standard at all. This scheme was of some temporary help to the tradesmen; but it was not long before someone found weights below zero again and the whole more-or-less system became unpopular among scientists although it is still used by a few practical groups.

It was all very confusing and the difficulties were of an astonishing variety, but we must leave to the imaginations of our readers the trials of the early scientists who tried to develop such concepts as mass, gravitation, and the laws of dynamics with a system including negative weights.

Eventually it became apparent that weight had an absolute value and the standards were themselves determined in terms of convenient units. The lighter stone was found to weigh about 273 convenient units, but as always, there were experimental errors, and various contemporaries used values ranging from 270 to 276 .

In due time the atomic theory was developed, and many years later the absolute weights of atoms were determined. For example, the deuterium atom was found to weigh $3.32 \times 10^{-24}$ convenient units; however, in accordance with an international agreement this result was recorded as $-272 \cdot 999,999,999,999,999,999,999,996,68 \pm 3 \cdot 0$ convenient units.

The principal difficulty in the system was that the ratio of the weights was obtained by adjusting their positions on a supporting beam and fulcrum and using the relation

$$
W_{0}=\frac{100}{\left(W_{1} / W_{0}\right)-1}
$$

The percentage error of $W_{1} / W_{0}$ was unfortunately multiplied by $3 \cdot 73$ in obtaining $W_{0}$. The limiting sensitivity of the balance was only one convenient unit of weight and one hesitates to contemplate the state of affairs if the standard stones had been 273 and 274 convenient units respectively. The experimenters would have obtained values of $W_{0}$ ranging from 137 to $\infty$.

Now it so happened that the pedestal on which the heavier standard was kept was not very resistant to acid fumes from the laboratory and it became weakened almost to the point of collapse. 